\section{IN THE NEWS}

Virtual bioinformatics Virtual reality will take on a whole new meaning following the award of $\mathrm{a} € 12$-million Commission of the European Union grant to create a pan-European Biosapiens Network of Excellence in Bioinformatics. The Network will create a virtual research institute to address the current fragmentation of European Bioinformatics. The European Molecular Biology Laboratory (EMBL) and the European

Bioinformatics Institute (EBI) informs us that scientists are currently "drowning in a sea of data" and that bioinformatics is struggling to keep up. Although Europe has excellent bioinformatics research institutes, according to Janet Thornton, Director of $\mathrm{EBI}$ and coordinator of the Biosapiens project, "to maximise the overall impact it [Europe] needs to strengthen and reinforce that excellence by restructuring and coordinating".

The European Union grant is part of the "Network of

Excellence", of which

"The Biosapiens project captures the most important objectives" (Janet Thornton). Not only will the Biosapiens project include a Virtual Institute for Genome Annotation, but the Institute will also establish a permanent European School of Bioinformatics. Professor Thornton believes that the project will indirectly benefit the exploitation of biological information to address issues such as health care, vaccines and personalized medicine.

The results of the work will be made freely available through one web portal. Because the project will make use of DAS (Distributed Annotation System), Søren

Brunak of the Technical University of Denmark believes that it "will herald a new era for database structure, where information is distributed by a network rather than a single site".
GENE EXPRESSION

\section{Revealing truths about non-coding transcripts}

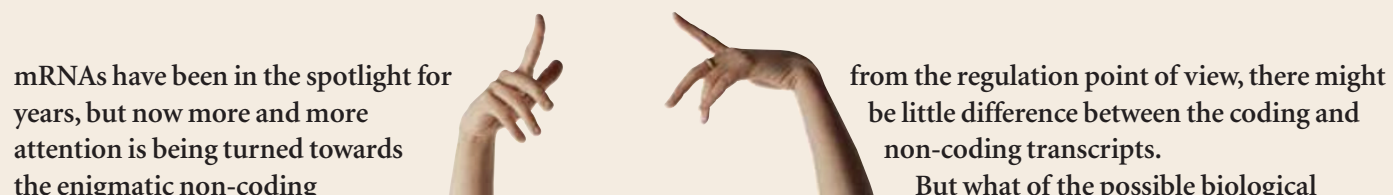
the enigmatic non-coding (nc)RNAs. Although computational and experimental approaches have identified some ncRNAs in several eukaryotic genomes, precious little is known about their function, let alone their regulation. A collaboration between the Struhl and Gingeras groups has just revealed that transcription of ncRNAs is regulated in a very similar way to that of coding RNAs - a finding that has important implications for our understanding of the biological function of these non-coding transcripts and of the transcriptome as a whole.

Comparisons between human and mouse genomes have releaved more than the expected number of conserved sequences, many of which lie far from annotated exons.

Moreover, previous studies have indicated that there is much more transcriptional activity in the genome than can be accounted for by current genome annotation. Wanting to explore the transcriptome and perhaps discover the missing transcripts, Cawley and Bekiranov et al. used a combination of high-density oligonucleotide arrays and chromatin immunoprecipitation to look for the binding sites of three well-known transcription factors: cMyc, Sp1 and p53. Their search, which was carried out in two cell lines, was restricted to the non-repeat genomic sequences of chromosomes 21 and 22.

Their results were unexpected - the number of transcription-factor binding sites was very large (ranging from 1,600 for p53 to 25,000 for cMyc, extrapolated to the whole genome) and their distribution was surprising. Only $22 \%$ of these sites lie within the $5^{\prime}$ termini of protein-coding genes, whereas $36 \%$ lie within or near the $3^{\prime}$ termini of protein-coding genes, indicating that they are distal regulatory elements or promoters for ncRNAs.

The possibility that these ncRNAs are bona fide transcripts was confirmed by RT-PCR - the sequences downstream of these binding sites are bone fide transcripts; moreover, many of them are polyadenylated. The observation that transcription factors that regulate the expression of protein-coding genes also regulate the transcription of ncRNAs is intriguing, for it opens up the possibility that, at least be little difference between the coding and

But what of the possible biological function of these non-coding transcripts? To investigate whether at least some of them are involved in differentiation, the authors monitored polyadenylated cytoplasmic RNAs in a plutipotent cell line as it was going through a programme of differention in response to retinoic acid (RA). Their observation that, both among the coding and non-coding transcripts, there was one population that was induced and one that was repressed in response to $\mathrm{RA}$ raises a couple of interesting points. First, non-coding transcription can be regulated in response to environmental signals. Second, what might the function of these non-coding transcripts be? If they are simply antisense molecules, the function of which is to regulate translation of their sense couterparts, why do they need to enter the cytoplasm at all if their task could be completed in the nucleus? Moreover, the authors saw coordinated expression of overlapping coding and non-coding transcripts in response to RA - contrary to what would be expected if the antisense ncRNAs were downregulating their coding counterparts. Might the non-coding transcripts function in the same pathway as the products of the coding genes?

Alternatively, the role of noncoding transcription might lie in the act of opening up the chromatin to facilitate access of trans-acting factors to the DNA.

Despite having focused only on two chromosomes, three transcription factors and one environmental induction condition, this study provides an unprecedented glimpse of the complexities of the transcriptome and its regulation. As is often the case, it raises more questions than it answers. In the light of the new findings, might we have to re-visit our definition of the gene or the role of transcription factors?

Magdalena Skipper

(9) References and links

ORIGINAL RESEARCH PAPER Cawley, S. \& Bekiranov, S, etal. Unbiased mapping of transcription factor binding sites along human chromosome 21 499-509 (2004)

FURTHER READING Mattick, J. S. RNA regulation: a new genetics. Nature Rev. Genet. 5, 316-323 (2004) and 22 points to widespread regulation of noncoding RNAs. Cell 116, 